\title{
PERCEPCIÓN DE RIESGO FRENTE AL COVID-19 EN ADOLESCENTES ESCOLARIZADOS COLOMBIANOS
}

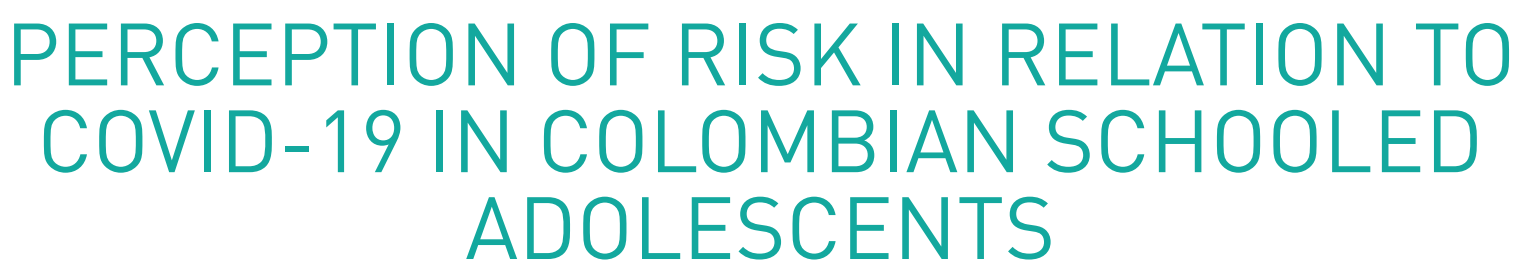

\author{
Aldayr David Rosero Bolaños ${ }^{1}$ \\ Jorge Luis Carvajal Guachavez ${ }^{2}$
}

Eduardo Fabio Bolaños Benavides ${ }^{3}$

\section{RESUMEN}

Se realizó un estudio cuyo objetivo fue determinar los niveles de la percepción de riesgo frente a la nueva enfermedad Covid-19 que presentó una muestra de 296 adolescentes escolarizados de la ciudad de Pasto - Colombia. El muestreo fue no probabilístico de tipo incidental. Se aplicaron de manera virtual dos instrumentos, que fueron un cuestionario socio económico y la escala: Percepción de Riesgo Frente al Covid-19 (PRFC), los cuales se sometieron a análisis estadísticos de tipo descriptivo- correlacional. 1 Psicólogo - Universidad de Nariño (Col), Docente Orientador - Colegio Colombo Suizo. davidbrs058@gmail. com. https://orcid.org/0000-0002-3451-1592

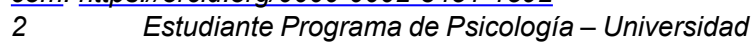
de Nariño (Col). JORGE LUIS CARVAJAL GUACHAVEZ https://orcid.org/0000-0001-9997-5307 gorgeluigi@hotmail.com

3 Estudiante Programa de Psicología - Universidad de Nariño (Col). EDUARDO FABIO BOLAÑOS eduardo9142@gmail.com

https://orcid.org/0000-0003-4019-4770
Los resultados muestran que la mayoría de participantes $(70 \%)$ presentan una percepción de riesgo alta y media frente a la enfermedad y sus consecuencias, destacándose el temor a la muerte de un familiar o a enfermar gravemente, también se identificó que los encuestados presentan una baja percepción de riesgo para los componentes relacionados con la probabilidad de contagiarse en situaciones de alta transmisión viral, por lo que no adoptan de manera adecuada las medidas de autocuidado y aislamiento social. Por lo anterior, se concluye que existe una tendencia a percibir el Covid-19 como una enfermedad que pone en riesgo la vida propia y de los demás, sin embargo, se presentan dificultades relacionadas con el desarrollo de hábitos que reduzcan la probabilidad de contagio del virus SARS-Cov-2, causante de la enfermedad ya mencionada. 


\section{PALABRAS CLAVE}

Percepción de riesgo, Adolescentes, Covid-19

\section{ABSTRACT}

A study was carried out to determine the levels of risk perception to the new disease Covid-19, was taken as a sample of 296 schooled adolescents from the city of Pasto - Colombia, the sampling was not probabilistic of incidental type. Two instruments were applied virtually, a socioeconomic questionnaire and the scale: Risk Perception of Covid-19 (PRFC), subjected to descriptive-correlational analysis. The results show most of the participants $(70 \%)$ present a high and medium risk perception of the disease and its consequences, emphasizing the fear about the death of a family member or of becoming seriously ill. Besides identified that the respondents present a low-risk perception for the components related to the probability of getting infected in situations of high viral transmission so that they do not adequately adopt the measures of self-care and social isolation. For the above mentioned, it's concluded there is a tendency to perceive Covid-19 as a disease that endangers one's own life and that of others; however, there are difficulties related to the development of habits that reduce the probability of contagion of SARS-Cov-2 virus, responsible for the abovemention disease.

\section{KEYWORDS}

Risk perception, Adolescents, Covid-19

\section{INTRODUCCIÓN}

La pandemia generada por el nuevo coronavirus SARS-CoV-2 ha seguido su curso, dejando una gran cantidad de víctimas mortales, sistemas sanitarios colapsados, crisis económicas en todos los países y la consolidación de nuevos hábitos como el teletrabajo, la educación virtual, el distanciamiento social y el uso de tapabocas para toda la población de manera obligatoria en el caso de Colombia (Otoya, García, Jaramillo \& Maecha, 2020).

Hasta el momento, los descubrimientos frente al comportamiento patógeno del virus SARS-Cov-2 y los medios de contagio han avanzado considerablemente, destacándose hallazgos como la posibilidad del agente viral de transmitirse vía aérea a través de aerosoles generados principalmente cuando la persona estornuda (Babu, Pandey \& Sheik, 2020) o la capacidad de supervivencia del virus en algunas superficies, alcanzando un máximo de hasta de dos días en material inerte y hasta de 3 horas en la piel humana (Badu, Pandey \& Sheik, 2020), siendo estos descubrimientos, la base para mejorar la comprensión de la transmisión de la enfermedad y permitiendo así establecer los mecanismos más apropiados para evitar el contagio.

De acuerdo a esto, diferentes organizaciones como por ejemplo la Organización Mundial de la Salud (OMS) y la Organización Panamericana de la Salud (OPS) han dispuesto diferentes tipos de medidas para ralentizar el virus con el fin de reducir el número de víctimas mortales y la saturación de las unidades de cuidados intensivos en los hospitales. Entre las medidas más eficaces se encuentran el lavado correcto de manos, el uso de tapabocas, la desinfección de superficies, la reducción del contacto físico entre personas, las restricciones para las reuniones sociales y la ventilación de espacios cerrados (Cruz, Santos, Cervantes \& Juárez, 2020; Gonzáles, 2020) conformando así, una serie de conductas individuales y sociales que cambian el panorama de la cotidianidad y chocan con las costumbres de toda la vida, generando la resistencia típica de los cambios de hábitos e imposibilitando el cumplimiento estricto de dichas recomendaciones (Lau, Griffiths, Choi \& Tsui, 2010).

De acuerdo a lo expuesto, el comportamiento de las personas y el cumplimiento de las normas 
y recomendaciones ya mencionadas, juega un papel fundamental frente a la evolución de la pandemia, donde entre mayores sean los cuidados individuales, menor será la tasa de contagio (Ochoa-Rosales, Gonzáles-Jaramillo, Vera-Calzaretta \& Franco, 2020; González, González, Gómez, Palacio, Gómez \& Franco, 2020), mitigando la saturación del sistema de salud y permitiendo el acceso a la atención médica para las personas con síntomas graves e indirectamente, evitando la toma de medidas restrictivas como lo es el confinamiento en casa o la declaración de cuarentena por parte de los gobiernos nacionales o locales.

Por lo anterior, los factores psicológicos y socio culturales relacionados con los comportamientos de las personas en torno a la percepción del riesgo de contagio de la enfermedad son un elemento que requiere ser estudiado con el fin de intervenir oportunamente en aquellas conductas y cogniciones que exponen en mayor medida a la persona y a la comunidad en general, tal como lo propone (Dammert-Elejalde,2020). De acuerdo a esto, disciplinas como la psicología y la sociología pueden generar diversos aportes que permitan comprender las razones por las cuales las personas no cumplen con las normas y restricciones de bioseguridad que se han impuesto, arriesgando la salud individual, familiar y comunitaria.

Son diversos los aportes que evidencian la importancia de la interdisciplinariedad para el abordaje delapandemia, porejemplo(CabanillasRojas, 2020; Estrella, 2020; Pérez \& Durán, 2020) exponen como programas educativos y de sensibilización han permitido generar una mayor adherencia a los cuidados frente al contagio y reducir el índice de los mismos. Por otra parte, (Dammert-Elejalde, 2020) expone que aspectos culturales, económicos y políticos tienen una fuerte incidencia sobre el cumplimiento de las normas para prevenir el contagio de COVID-19, donde se ha descubierto que países con mayor calidad educativa y mejores índices de cultura ciudadana, son los que cumplen de mejor manera los lineamientos para hacer frente a la pandemia.

Por lo anterior, la comprensión de elementos como la percepción de riesgo son fundamentales para identificar aquellos aspectos que requieren intervenirse para mejorar la adopción de comportamientos orientados a prevenir el contagio por el nuevo coronavirus. En relación a esto, Zimolong y Trimpop (2012), definen la percepción de riesgo como la evaluación cognitiva que genera una determinación del nivel de peligro de una situación; adicional a ello, los investigadores identificaron que los estímulos que tienen una mayor percepción del riesgo se caracterizan por ser novedosos, repentinos o de gran intensidad.

Por su parte, Morillejo, Pozo y Martínez (2012) complementan el concepto de percepción de riesgo considerando que este proceso psicológico no solo se reduce a elementos cognitivos, sino que también constituye un factor desencadenante de determinadas conductas que se generan ante diferentes situaciones, según el potencial de peligro que interpreta una persona.

Si bien, no es posible encontrar investigaciones similares al presente estudio, existen algunos antecedentes donde se evalúan las percepciones de riesgo frente a enfermedades pandémicas. Por ejemplo, Agüero, Nebot, Pérez, López y García, (2011), identificaron características sobre la percepción de riesgo de jóvenes y adultos españoles durante la pandemia de H1N1 en el año 2009, destacándose en la muestra, una alta sensación de riesgo a contagiarse del virus y un ajuste moderado a los comportamientos de autocuidado recomendados por las autoridades sanitarias. Adicional a este estudio, se han desarrollado diferentes investigaciones sobre la percepción de riesgo frente a infecciones de transmisión sexual en especial al $\mathrm{VIH}$, 
donde se encontró que los jóvenes tienden a minimizar el peligro que conduce el contagio debido a factores como el tiempo que transcurre entre la infección y los síntomas, además de presentarse diferencias significativas según el nivel educativo y aspectos culturales como el país donde se desarrollaron los estudios, siendo los jóvenes residentes en África y Latinoamérica los más vulnerables frente a esta enfermedad (Saura, Jorquera, Rodríguez, Mascort, Castella \& García, 2019; Gonzáles, 2019; Rosabal, Sánchez, Roabal, Arias \& Rodríguez, 2016).

Los antecedentes sobre la percepción de riesgos frente a enfermedades infecciosas muestran la existencia de diferentes modelos teóricos que abordan esta temática, sin embargo, el modelo de Creencias de la Salud (MCS) propuesto por Rosentock (1974) citado en del Castillo (2012) se ha consolidado como uno de los mejores al momento de estudiar los comportamientos y generar predicciones relacionadas con la conducta y las implicaciones que esta tiene en el bienestar de las personas en contextos de riesgo (del Castillo, 2012). En cuanto al modelo MCS se puede evidenciar que este se centra en la evaluación de la ejecución de conductas específicas que se encuentran mediadas por la incertidumbre o el miedo, considerando adecuados, aquellos comportamientos de evitación frente a los diferentes riesgos para la salud, y contemplando los comportamientos de protección (del Castillo, 2012).

Acorde a lo anterior, una percepción de riesgo adecuada se configura a través de la interacción de diferentes elementos que orientan a una persona hacia un comportamiento saludable, destacándose variables como la motivación por mantenerse sano, la calidad de la información que posee la persona para su cuidado y la estructura de las creencias personales y sociales (Medina \& León, 2004; Valencia, Londoño, Amézquita, Cortés, Guerra, Hurtado \& Ordoñez, 2009).
Teniendo en cuenta lo expuesto y con el fin de delimitar aquellos aspectos relacionados con la percepción de riesgo según el modelo MCS, se utilizarán los componentes propuestos por Green, Murphy y Gryboski (2020), quienes afirman que existen tres factores que permiten evaluar la percepción de riesgo; en primer lugar se encuentra la evaluación que realiza la persona frente a la susceptibilidad o vulnerabilidad de enfermar, seguido por la gravedad percibida de las consecuencias en caso de enfermar o que un familiar o ser querido enferme, por último, está la evaluación de los costes y beneficios de la adopción de conductas saludables y que reducen las barreras psicológicas, físicas y sociales que maximizan el riesgo (del Castillo, 2012).

Sin duda alguna, la comprensión de elementos psicosociales como la percepción de riesgo frente al contagio del nuevo coronavirus constituyen un cuerpo teórico importante para abordar el modelo de alternancia propuesto por el Ministerio de Educación de Colombia, en el cual se establecen lineamientos para la prestación del servicio educativo de manera presencial y virtual (Ministerio de Educación Nacional, 2020).

Teniendo en cuenta lo expuesto, el objetivo del presente estudio es determinar los niveles y algunas características de las percepciones de riesgo frente al contagio del nuevo coronavirus SARS-CoV-2 y sus posibles consecuencias, en una muestra de adolescentes escolarizados de la ciudad de San Juan de Pasto - Colombia.

\section{MÉTODO}

\section{Participantes}

El presente estudio contó con una muestra tipo incidental, la cual estuvo conformada por 296 estudiantes de 5 instituciones educativas de la ciudad de Pasto- Colombia. En cuanto a las características socio demográficas 
de los adolescentes, se encontró que sus edades oscilaban entre los 13 a los 18 años $(M=15,47 ; S D=1,29)$ quienes actualmente cursan los grados octavo a undécimo de bachillerato. El 50,3\% fueron mujeres $(49,7 \%$ hombres), los cuales pertenecen a los estratos socioeconómicos 2 a 5 .

\section{INSTRUMENTOS}

En primer lugar, se presentó un cuestionario socio económico que permitió determinar el sexo, la edad, el grado de escolaridad y el estrato socio económico de los estudiantes.

A continuación, se presentó un cuestionario titulado "Percepción de Riesgo Frente al Covid-19" (PRFC) de elaboración propia, el cual se estructuró con 21 ítems que se califican de 1 a 4 (nada probable $=1$, poco probable $=2$, probable $=3$, muy probable $=4$ ) y se organiza en 3 factores que son: Susceptibilidad de enfermar; compuesto por 10 ítems, gravedad percibida en caso de enfermar; compuesto por 6 ítems y percepción frente a conductas protectoras; compuesto por 5 ítems. Cabe mencionar que los ítems 10,19 y 20 se califican de manera inversa.

Este instrumento que determina características de los factores de riesgo frente al contagio del nuevo coronavirus (SARS-CoV-2) y los efectos indirectos que este pueda tener en el entorno cercano de los participantes fue sometido a un proceso de pilotaje para realizar ajustes lingüísticos, posteriormente se realizaron análisis estadísticos en los cuales el test mostró propiedades psicométricas adecuadas, con una fiabilidad alta para todos sus factores y la escala general ( $\alpha$ factor $1=0.92$; $\alpha$ factor $2=$ 0.82 ; $\alpha$ factor $3=0.83$; $\alpha$ total $=0.86$ ), además de mostrar índices de ajustes adecuados para la distribución factorial propuesta por los autores: Prueba KMO: 0.903; Esfericidad de Barlett: $p$ $<0.05$.

\section{PROCEDIMIENTO}

El proceso de reclutamiento de participantes y la recolección de datos en general se realizó de manera virtual, utilizando dos vías electrónicas que fueron el correo electrónico y la aplicación móvil de mensajería Whatsapp.

A través de las vías de comunicación ya mencionadas, se brindó toda la información necesaria para explicar los objetivos de este estudio, aclarar dudas y establecer el compromiso para la devolución de emergentes.

El diligenciamiento de los cuestionarios y la firma del consentimiento informado y consentimiento asistido se realizaron dentro del mismo formulario del online utilizado para recolectar la información que para esta ocasión fue Google Forms.

Por último, se realizó un vaciado y depuración de la información recolectada a través del software Excel para su posterior análisis.

\section{ELEMENTOS ÉTICOS Y BIOÉTICOS}

La presente investigación y sus subprocesos se fundamentaron en los principios éticos contemplados en el código Deontológico y Bioético de Psicólogo en Colombia, Ley 1090 de 2006, junto con esto se tuvo en cuenta los lineamientos establecidos por la Resolución 8430 de 1993 por la cual se dictan las normas científicas, técnicas y administrativas para una investigación en salud en Colombia, especialmente en su título II "de la investigación en seres humanos". Se brindó de manera virtual toda la información necesaria a los participantes y sus acudientes, además de hacer explícito el objetivo del estudio, los riesgos y beneficios del mismo, por otra parte, se enfatizó sobre el carácter anónimo, confidencial y voluntario de su participación y la posibilidad de retirarse del estudio en el momento que el participante o su tutor lo considere. 


\section{ANÁLISIS DE DATOS}

Los análisis estadísticos se llevaron a cabo con el software SPSS.23 (IBM Corp., 2015). En primer lugar, se analizó el cuestionario socioeconómico aplicado a los estudiantes donde se calcularon las frecuencias, promedio y desviación estándar para para la variable edad y las frecuencias y porcentajes de las variables sexo, nivel de escolaridad y estrato socioeconómico.

A continuación, se calculó el promedio, el rango y la desviación estándar de los puntajes de cada ítem, subescala y la escala total del cuestionario PRFC, además se determinó la asociación entre las variables sexo, edad, estrato socio económico y nivel educativo a través de una prueba Rho Spearman.

El nivel de significancia adoptado en los análisis correlacionales fue: $p<0,05$.

Por último, se obtuvo los percentiles según el puntaje total de la escala PRFC con el fin de determinar de forma exploratoria la distribución de la muestra según la percepción de riesgo encontrada en la evaluación, se establecieron los siguientes puntos de corte: Percentiles 70 a
100: percepción de riesgo alta; percentiles 60 a 40: percepción de riesgo medio; percentiles 30 a 10: percepción de riesgo baja.

\section{RESULTADOS}

En términos generales, la mayoría de respuestas de los participantes se encuentran en las opciones: probable y muy probable de la prueba PRFC, lo cual indica que gran parte de los estudiantes que conformaron la muestra perciben los diferentes riesgos y consecuencias de contraer la enfermedad Covid-19 como eventos probables.

La media de respuesta en general fue de 2,91 con una desviación estándar de 0,47 , evidenciando que la tendencia de respuesta del presente estudio fue la opción probable. El análisis descriptivo por ítems (Ver tabla 1) muestra que la pregunta 12 que evalúa la percepción de la gravedad en caso de que un familiar enferme, tuvo el promedio más alto $(\mathrm{M}=3,34)$, seguido del ítem 17 que hace referencia a la percepción frente a la probabilidad de que un familiar muera. Lo anterior, muestra que los participantes perciben un mayor riesgo ante la gravedad de la enfermedad Covid-19, especialmente si la contrae un familiar.

Tabla 1.

Descriptivos por ítem - Prueba PRFC

\begin{tabular}{|c|l|c|c|c|c|c|c|}
\hline No. & \multicolumn{1}{|c|}{ Ítem } & $\begin{array}{c}\text { Nada } \\
\text { probable } \\
(\%)\end{array}$ & $\begin{array}{c}\text { Poco } \\
\text { probable } \\
(\%)\end{array}$ & $\begin{array}{c}\text { Probable } \\
(\%)\end{array}$ & $\begin{array}{c}\text { Muy } \\
\text { probable } \\
(\%)\end{array}$ & Media & $\begin{array}{c}\text { Desviación } \\
\text { estándar }\end{array}$ \\
\hline 1. & $\begin{array}{l}\text { ¿Qué tan probable } \\
\text { es contagiarse de } \\
\text { Covid-19 por no usar } \\
\text { tapabocas? }\end{array}$ & 4,7 & 10,1 & 70,9 & 14,2 & 2,95 &, 656 \\
\hline 2. & $\begin{array}{l}\text { ¿Qué tan probable } \\
\text { es contagiarse de } \\
\text { Covid-19 por asistir a } \\
\text { reuniones familiares } \\
\text { o de amigos? }\end{array}$ & 13,7 & 7,1 & 61,1 & 18 & 2,14 &, 695 \\
\hline
\end{tabular}




\begin{tabular}{|c|c|c|c|c|c|c|c|}
\hline 3. & $\begin{array}{l}\text { ¿Qué tan probable } \\
\text { es contagiarse } \\
\text { de Covid-19 por } \\
\text { tocarse la cara } \\
\text { constantemente? }\end{array}$ & 15,4 & 7,1 & 48,4 & 29,1 & 2,31 & 753, \\
\hline 4. & $\begin{array}{l}\text { ¿Qué tan probable } \\
\text { es contagiarse de } \\
\text { Covid-19 por no } \\
\text { lavarse las manos } \\
\text { continuamente? }\end{array}$ & 3,7 & 8,8 & 56,1 & 31,4 & 3,15 & ,728 \\
\hline 5. & $\begin{array}{l}\text { ¿Qué tan probable } \\
\text { es contagiarse de } \\
\text { Covid-19 al tener } \\
\text { contacto físico con } \\
\text { una persona que ha } \\
\text { adquirido el virus? }\end{array}$ & 4,1 & 10,8 & 60,5 & 24,7 & 3,06 & ,718 \\
\hline 6. & $\begin{array}{l}\text { ¿Qué tan probable } \\
\text { es contagiarse de } \\
\text { Covid-19 cuando se } \\
\text { habla de cerca con } \\
\text { una persona que no } \\
\text { usa tapabocas? }\end{array}$ & 7,4 & 10,5 & 55,7 & 26,4 & 3,01 & 817, \\
\hline 7. & $\begin{array}{l}\text { ¿Qué tan probable } \\
\text { es contagiarse } \\
\text { de Covid-19 por } \\
\text { encontrarse en } \\
\text { lugares concurridos y } \\
\text { poco ventilados? }\end{array}$ & 5,7 & 18,8 & 48,1 & 27,4 & 2,77 & ,767 \\
\hline 8. & $\begin{array}{l}\text { ¿Es probable } \\
\text { contagiarse de } \\
\text { Covid-19 por tener } \\
\text { contacto con una } \\
\text { persona que no } \\
\text { presenta síntomas? }\end{array}$ & 14,4 & 11,8 & 45,7 & 28 & 2,17 & ,755 \\
\hline 9. & $\begin{array}{l}\text { ¿Qué tan probable } \\
\text { es contagiarse de } \\
\text { Covid-19 por asistir } \\
\text { a eventos masivos } \\
\text { como celebraciones, } \\
\text { fiestas, conciertos, } \\
\text { etc.? }\end{array}$ & 4,7 & 10,5 & 60,5 & 24,3 & 3,04 & ,733 \\
\hline
\end{tabular}




\begin{tabular}{|c|c|c|c|c|c|c|c|}
\hline 10. & $\begin{array}{l}\text { ¿Qué tan probable } \\
\text { podría ser un } \\
\text { contagio de Covid-19 } \\
\text { si sigo al pie de la } \\
\text { letra los protocolos } \\
\text { de bioseguridad } \\
\text { (desinfección de } \\
\text { manos y zapatos, } \\
\text { mantener distancia } \\
\text { de otras personas)? }{ }^{*}\end{array}$ & 6,1 & 8,1 & 53,4 & 32,4 & 3,12 & ,797 \\
\hline 11. & $\begin{array}{l}\text { ¿Qué tan probable } \\
\text { es que muera por } \\
\text { contagiarme con } \\
\text { Covid-19? }\end{array}$ & 6,8 & 8,8 & 27 & 57,4 & 3,25 & ,901 \\
\hline 12. & $\begin{array}{l}\text { ¿Qué tan probable } \\
\text { es que sea } \\
\text { hospitalizado por } \\
\text { contraer Covid-19? }\end{array}$ & 5,7 & 8,8 & 31,4 & 54,1 & 3,34 & ,864 \\
\hline 13. & $\begin{array}{l}\text { ¿Qué tan probable } \\
\text { es tener secuelas o } \\
\text { problemas de salud } \\
\text { permanentes tras } \\
\text { haberme contagiado } \\
\text { de Covid-19? }\end{array}$ & 7,1 & 11,1 & 31,4 & 50,3 & 3,25 & ,916 \\
\hline 14. & $\begin{array}{l}\text { ¿Qué tan probable } \\
\text { es presentar } \\
\text { síntomas } \\
\text { respiratorios graves } \\
\text { por infectarme con } \\
\text { Covid-19? }\end{array}$ & 7,1 & 8,8 & 36,1 & 48 & 3,25 & ,890 \\
\hline 15. & $\begin{array}{l}\text { ¿Qué tan probable } \\
\text { es que algún familiar } \\
\text { cercano muera por } \\
\text { contagiarse con } \\
\text { Covid-19? }\end{array}$ & 5,4 & 8,1 & 41,2 & 45,3 & 3,26 & ,826 \\
\hline 16. & $\begin{array}{l}\text { ¿Qué tan probable } \\
\text { es que algún } \\
\text { familiar cercano sea } \\
\text { hospitalizado por } \\
\text { contraer Covid-19? }\end{array}$ & 6,1 & 11,5 & 34,1 & 48,3 & 3,25 & ,885 \\
\hline
\end{tabular}




\begin{tabular}{|c|l|c|c|c|c|c|c|}
\hline 17. & $\begin{array}{l}\text { ¿Qué tan probable } \\
\text { es que utilice el } \\
\text { tapabocas para } \\
\text { evitar que mi familia } \\
\text { se infecte? }\end{array}$ & 4,1 & 12,5 & 30,7 & 52,7 & 3,32 &, 845 \\
\hline 18. & $\begin{array}{l}\text { ¿Qué tan probable } \\
\text { es que siga los } \\
\text { protocolos de } \\
\text { bioseguridad de mi } \\
\text { colegio para que no } \\
\text { haya más contagios? }\end{array}$ & 5,4 & 12,8 & 27,4 & 54,4 & 3,31 &, 893 \\
\hline 19. & $\begin{array}{l}\text { ¿Qué tan probable } \\
\text { es que no use el } \\
\text { tapabocas por } \\
\text { pereza u olvido? * }\end{array}$ & 18,4 & 17,6 & 28,7 & 35,3 & 2,81 &, 979 \\
\hline 20. & $\begin{array}{l}\text { ¿Cuál es la } \\
\text { probabilidad de que } \\
\text { no asista a fiestas y } \\
\text { reuniones por temor } \\
\text { a infectar a otras } \\
\text { personas? }\end{array}$ & 15,4 & 24,5 & 21,8 & 38,3 & 3,17 &, 891 \\
\hline 21. & $\begin{array}{l}\text { ¿Qué tan } \\
\text { probable es que } \\
\text { lave mis manos } \\
\text { frecuentemente, } \\
\text { aunque sienta } \\
\text { pereza de hacerlo? }\end{array}$ & 24,4 & 10,5 & 34,5 & 30,7 & 2,01 &, 831 \\
\hline
\end{tabular}

Fuente: Elaboración propia * Ítems que se califican de manera inversa

Se destacan también los promedios altos de los ítems 11, 13 y 14, los cuales miden la percepción de riesgo de enfermar con gravedad o morir en caso de contraer el virus causante del Covid-19. Lo anterior muestra que la percepción de riesgo de los participantes está orientada principalmente a las consecuencias que la enfermedad puede generar, por encima de las conductas de prevención y los riesgos en los posibles escenarios de contagio del virus.

De acuerdo a lo anterior, se encontraron ítems con promedios inferiores a 3 , donde se destaca el ítem 21: "¿Qué tan probable es que lave mis manos frecuentemente, aunque sienta pereza de hacerlo?", evidenciándose una situación similar en el ítem 19: “¿Qué tan probable es que no use el tapabocas por pereza u olvido?" lo cual indica que las medidas de autocuidado pueden estar mediadas de manera negativa por factores individuales y sociales que reducen la frecuencia de emisión de estas conductas protectoras,.

Por otra parte, se evidencian diversos ítems con promedios bajos en el factor Susceptibilidad de enfermar, destacándose los ítems 2, 7 y 8 , referentes al contacto social o a la exposición de contagio en lugares concurridos. Sumado a lo anterior, llama la atención los bajos promedios de las preguntas 1 y 3 , las cuales se relacionan con la relación entre la percepción del riesgo de contraer el virus, con comportamientos como el 
uso de tapabocas o tocarse la cara.

El análisis descriptivo de los factores de la prueba PRFC (Ver tabla 2) indica rangos amplios en todos los componentes, lo cual evidencia la presencia de participantes que consideran improbable diversos aspectos como la probabilidad de contagiarse o seguir las diferentes medidas de bioseguridad.

Tabla 2.

Estadísticos descriptivos por factores

\begin{tabular}{|l|c|c|c|c|}
\hline \multicolumn{1}{|c|}{ Factor Prueba PRFC } & Mínimo & Máximo & Media & $\begin{array}{c}\text { Desviación } \\
\text { estándar }\end{array}$ \\
\hline Susceptibilidad de enfermar & 10,00 & 40,00 & 30,7 & 5,73 \\
\hline $\begin{array}{l}\text { Gravedad percibida en caso de } \\
\text { enfermar }\end{array}$ & 6,00 & 24,00 & 19,6 & 3,86 \\
\hline $\begin{array}{l}\text { Percepción frente a conductas } \\
\text { protectoras }\end{array}$ & 5,00 & 20,00 & 16,2 & 3,34 \\
\hline Total & 30,00 & 84,00 & 66,7 & 8,99 \\
\hline
\end{tabular}

Fuente: Elaboración propia

Los promedios encontrados en el presente análisis indican un ajuste adecuado en todos los componentes, sin embargo, se observa que el primer factor muestra una media más baja si se tiene en cuenta el número de ítems que lo componen, por lo que se puede considerar que un grupo de participantes tienden a tener una percepción de riesgo baja frente a la posibilidad de contagiarse, lo que se refleja en un limitado ajuste a las normas de bioseguridad como por ejemplo el uso de tapabocas o limitar el contacto social.
En cuanto a los análisis inferenciales, no se encontraron correlaciones estadísticamente significativas entre las variables socio demográficas y el nivel educativo con la prueba PRFC y sus sub escalas, mostrando índices Rho menores a 0,30 y valores $p>0,05$.

A modo de complemento, se obtuvo los percentiles con respecto al puntaje final de la prueba PRFC (Ver tabla 3).

Tabla 3.

Percentiles - Puntaje Total Prueba PRFC

\begin{tabular}{ccccccccccc}
\hline Percentil & 10 & 20 & 30 & 40 & 50 & 60 & 70 & 80 & 90 \\
\hline $\begin{array}{c}\text { Puntaje de } \\
\text { referencia }\end{array}$ & $56-$ & $60-$ & 62 & 65 & 68 & 66 & 71 & 73 & 75 & 84 \\
$\begin{array}{c}\text { Número de } \\
\text { casos }\end{array}$ & 36 & 27 & 28 & 27 & 43 & 37 & 34 & 33 & 31 \\
\hline
\end{tabular}

Fuente: Elaboración propia 
Se observa que cerca del $33,1 \%$ de participantes se encuentran dentro de los percentiles con puntajes que se pueden interpretar como una percepción de riesgo alta frente a la posibilidad de contagio de la enfermedad Covid-19, como de las diferentes consecuencias que esta afección puede generar.

Se encuentra que el $36,2 \%$ de participantes muestran una percepción de riesgo medio frente a los factores evaluados por el cuestionario PRFC.

Por otra parte, se evidencia que 91 participantes $(30,7 \%)$ se encuentran dentro de los percentiles 10 a 30 , los cuales pueden interpretarse como una percepción de riesgo baja, evidenciando un escaso apego a las normas de bioseguridad como a las diferentes conductas de autocuidado frente al nuevo coronavirus.

\section{DISCUSIÓN}

En cuanto a los resultados del presente estudio, se encuentra que el $70 \%$ de participantes posee una percepción del riesgo media o alta, lo cual puede reflejarse en la adopción de las diferentes medidas de bioseguridad y las conductas de autocuidado, lo cual puede deberse a diferentes factores que pueden ser el temor a morir o enfermar gravemente o que esta situación se presente en un familiar cercano, situación que se corroboró en el análisis por ítem de la prueba PRFC, donde sobresalieron preguntas relacionadas con la gravedad de la enfermedad Covid-19 o la probabilidad de morir por esta patología.

Aunque la comparación con otros estudios es limitada debido a la escasa información disponible y lo novedoso del fenómeno, se logró determinar que los resultados de esta investigación guardan similitudes importantes con el estudio realizado por Agüero et al. (2011) en España durante la pandemia generada por el virus $\mathrm{H} 1 \mathrm{~N} 1$, donde el $77 \%$ de participantes consideraba a la enfermedad y la posibilidad de contagio como un riesgo significativo, el cual debía afrontarse utilizando al menos un mecanismo de prevención o autocuidado. Este contraste muestra una tendencia tanto en la pandemia del 2009 causada por el H1N1, como la que se viene desarrollando en la actualidad a causa del nuevo coronavirus SARS-CoV-2, en donde un segmento de las muestras cercano al $30 \%$ perciben un riesgo bajo frente a estas enfermedades, afectando negativamente la adopción de las diferentes medidas de bioseguridad.

En cuanto a los hallazgos encontrados en los análisis de ítems, se tiene que los reactivos con promedios más altos hacen referencia a aspectos relacionados con las consecuencias de contraer el virus causante del Covid-19, especialmente con la percepción de los participantes frente a la probabilidad de que un familiar o ellos mismos mueran a causa de esta afección, donde el $54 \%$ consideran que estas situaciones tienen una alta probabilidad de que sucedan. Los resultados obtenidos en este estudio son similares a los encontrados por De los Ríos, Gutiérrez, Cuno y Llanos (2020), quienes estudiaron los diferentes temores durante la cuarentena en una muestra de adultos en Perú, evidenciando que el $49 \%$ de su muestra presenta un temor constante de que un familiar o si mismos mueran.

Lo anterior, ha sido estudiado de manera exploratoria por diferentes autores como por ejemplo Muñoz (2020); que consideran que el temor a infectarse o a morir son aspectos que deben ser atendidos de manera prioritaria debido a las múltiples afectaciones en la salud mental que estas cogniciones pueden generar, siendo la ansiedad y la depresión los principales trastornos que se configuran en torno a estos pensamientos.

Por otra parte, se tiene que los ítems con promedios más bajos están relacionados con conductas de autocuidado como el uso de 
tapabocas o el distanciamiento social, siendo estos aspectos, los más infravalorados por los participantes. Lo anterior se traduce en una baja percepción de riesgo frente a contagiarse del nuevo coronavirus si no se toman las medidas recomendadas por las autoridades de salud y el gobierno nacional.

La anterior situación pone en evidencia una tendencia que parece desarrollarse en el contexto latinoamericano, donde se han encontrado cifras similares a las del presente estudio. A modo de ejemplo, se destaca una investigación realizada en México con una muestra amplia, donde el $22 \%$ de participantes reportó no lavar sus manos frecuentemente, mientras que un $34 \%$ no considera relevante mantener el distanciamiento recomendado (Galindo-Vázquez, Ramírez-Orozco, CostasMuñiz, Mendoza-Contreras, Calderillo-Ruíz \& Meneses-García, 2020); cifras que son muy parecidas a las encontradas en un estudio desarrollado con jóvenes de Perú, Argentina y Colombia, donde el 35\% reportó no mantener el distanciamiento social recomendado y un $20 \%$ manifestó no haber respetado el aislamiento con el fin de mantener contacto social (Diez et al., 2020).

Con relación a los análisis descriptivos realizados a los factores de la prueba PRFC, se tiene que la percepción de riesgo que tienen los participantes se orienta principalmente a las consecuencias que puede generar la enfermedad Covid-19, dejando de lado aspectos como las conductas de autocuidado o minimizando las posibilidades de contagio frente a situaciones especificas como por ejemplo asistir a reuniones o tener contacto con personas sin el uso de tapabocas $\mathrm{u}$ otros cuidados.

Como ya se ha expuesto anteriormente, las diferentes investigaciones evidencian que la mayoría de jóvenes perciben de manera adecuada los diferentes riesgos que podría generar tanto para si mismos como para sus familiares un contagio por el nuevo coronavirus SARS-CoV-2, sin embargo, un segmento de las diferentes muestras estudiadas, incluida la presente; indican que el riesgo percibido para el Covid-19 se encuentra fuertemente dirigido a las consecuencias, restando importancia a aspectos relacionados a las causas de contagio y la aplicación de comportamientos para prevenirlo.

Sin embargo, esta tendencia parece no ser ajena a la pandemia que se vive actualmente, Aragonés, Talayero y Olivos (2010) determinaron que durante la crisis sanitaria generada por el virus H1N1, las personas dieron mayor preponderancia a aspectos catastróficos y el riesgo de muerte, por encima de los hábitos de higiene y otros elementos de autocuidado recomendados en ese momento.

Estos hallazgos indican una posible constante en los comportamientos y cogniciones de las personas frente a situaciones referentes a las crisis sanitarias, donde el factor común es el temor a la muerte o a enfermar gravemente, elementos que se relacionan directamente con el instinto humano de supervivencia, sin embargo, esto puede generar indirectamente la omisión de aquellos comportamientos que conllevan a reducir las probabilidades de contagio, generando una más alta vulnerabilidad en aquellos segmentos poblacionales que tienen una percepción de riesgo baja o nula con respecto a la posibilidad de contraer el virus causante del Covid-19.

Por otra parte, aunque el presente estudio no mostródiferenciasestadísticamentesignificativas al comparar los niveles de percepción de riesgo frente al Covid-19, teniendo en cuenta diferentes variables socio demográficas como la edad, el sexo y el estrato socio económico de los participantes, los diferentes estudios indican que elementos como el nivel educativo, el acceso a los sistemas de salud y el estrato social juega un papel fundamental en los índices de contagio, la adherencia a los comportamientos de 
autocuidado y el riesgo de muerte, donde entre menor sea el índice educacional, los ingresos familiares y otros factores relacionados, mayor será la probabilidad de contraer el nuevo coronavirus (Ortiz-Hernández \& Pérez-Sastré, 2020).

Por lo anterior, se puede pensar que, por debido al tamaño y la naturaleza de la muestra del presente estudio y otros elementos como la edad de los participantes, no es posible evidenciar las diferencias ya mencionadas, que se han encontrado en investigaciones que abarcan muestras mucho más grandes y heterogéneas.

A modo de reflexión, se puede afirmar que, aunque los datos encontrados en este estudio son similares a otras investigaciones, se requieren mayores esfuerzos por parte de los diferentes actores involucrados en los procesos educativos de los niños y adolescentes con el fin de fortalecer aquellas dimensiones referentes al autocuidado y sensibilizar a esta población frente a la necesidad de ajustarse a las recomendaciones de las autoridades sanitarias para reducir los diferentes riesgos que puede traer la implementación del modelo de alternancia en todos los niveles educativos.

Acorde a lo anterior, existe evidencia empírica que muestra la posibilidad de mejorar el ajuste - la adherencia a aquellos nuevos hábitos como el uso del tapabocas, el aislamiento social o la evitación a la asistencia de eventos y aglomeraciones. Dentro de la literatura científica, se destacan las intervenciones y acciones propuestas por Kouhpayeh, Jeihooni, Kashfi y Bahmandoost (2017) y Arteaga (2020) quienes evidencian la posibilidad de optimizar diversos aspectos relacionados con las percepciones de riesgo frente a enfermedades infecciosas desde una perspectiva interdisciplinaria que articula acciones micro contextuales como lo es la psicoeducación en el aula, como también intervenciones de carácter macro contextual como por ejemplo el uso de TICs (televisión y redes sociales) y el desarrollo de programas de apoyo estatal que garanticen el apoyo económico y técnico necesario.

\section{CONCLUSIONES}

Los resultados del presente estudio permiten concluir que existe una percepción de riesgo frente a la posibilidad de contagio del Covid-19 y de sus consecuencias en un nivel alto y medio en cerca del $70 \%$ de participantes de la muestra, mientras que el $30 \%$ restante, ha minimizado los diferentes riesgos relacionados con esta enfermedad pandémica.

Los elementos que los participantes perciben con mayor riesgo hacen referencia a las posibilidades de morir, ser hospitalizado o presentar secuelas después de infectarse por el nuevo coronavirus, además de presentarse una percepción de riesgo alta ante la posibilidad de que un familiar muera o enferme gravemente.

Contrario a lo anterior, los adolescentes encuestados presentan una baja percepción de riesgo ante las posibilidades de contagiarse de la enfermedad Covid-19 en situaciones relacionadas con el contacto social y el desconocimiento del riesgo de contagio al tener contacto con personas asintomáticas.

Sumado a lo expuesto, se identificó que existe una percepción del riesgo inadecuada en relación a las conductas de autocuidado, donde un segmento de participantes muestra una baja adherencia a conductas de autocuidado como por ejemplo el uso de tapabocas y el lavado de manos.

\section{RECOMENDACIONES}

Se recomienda realizar estudios similares con muestras más amplias y heterogéneas, estipulando la posibilidad de reclutar participantes de diferentes regiones del territorio nacional con el fin de identificar diferencias socio culturales. 
El instrumento PRFC utilizado en la presente investigación puede constituir un elemento de medición y evaluación importante para identificar las fortalezas y debilidades de la población estudiantil con el fin de preparar intervenciones y acciones psicoeducativas de cara al inicio del modelo de alternancia, por lo que se recomienda su uso en muestras de adolescentes.

Es recomendable adaptar el instrumento PRFC para la población escolar infantil con el fin de permitir realizar mediciones en los diferentes niveles educativos obligatorios.

\section{CONFLICTO DE INTERESES}

El autor/es de este trabajo declaran que no existe conflicto de intereses

\section{FINANCIACIÓN}

El presente estudio se realizó con recursos propios.

\section{REFERENCIAS BIBLIOGRÁFICAS}

Agüero, F., Nebot, M., Pérez, A., López, M. J., \& García, X. (2011). Actitudes y comportamientos preventivos durante la pandemia de gripe (H1N1) 2009 en España. Revista española de salud pública, 85(1), 73-80. Recuperado en 30 de noviembre de 2020 , de

http://scielo.isciii.es/scielo. php?script=sci_arttext $\&$ pid $=$ S1135$57272011000100009 \&$ Ing=es\&tlng=es.

Aragonés, J. I., Talayero, F., \& Olivos, P. (2010). Percepción del riesgo de la gripe A (H1N1) desde el "paradigma psicométrico". Revista de Psicología Social, 25(3), 271-282.

https://doi.org/10.

1174/021347410792675633
Arteaga, N. L. C. (2020). La política cubana frente a la covid19. La educación en este contexto. Revista Boletín Redipe, Vol. 9 $N^{\circ} 8,2020$. Pags. 24-34. https://doi.org/10.36260/rbr.v9i8.1038

Babu, S., Pandey, D., \& Sheik, I. (2020). Acts Of COVID19. International Journal of Advanced Engineering, 3, 2457-0397.

Cabanillas-Rojas, W. (2020) Conducta Y Propagación Del Covid-19 En El Perú: Marco De Referencia Para El Diseño De Intervenciones Conductuales De Salud Pública. [Preprint] https://doi. org/10.1590/SciELOPreprints.868

Cruz, M. P., Santos, E., Cervantes, M. V., \& Juárez, M. L. (2020). COVID-19, una emergencia de salud pública mundial. Revista Clínica Española.

Dammert-Elejalde, R. (2020). COVID-19: Influencias Socio Culturales $Y$ Adherencia A Las Estrategias Gubernamentales De Aislamiento Social En El Perú. Revista De La Facultad De Medicina Humana, 20(4), 3-3. https://doi.org/ 10.25176/RFMH.v20i4.3044

de los Ríos, L. R., Gutiérrez, R., Cuno, R. Q., \& Llanos, Y. M. C. (2020). Covid-19: Frecuencia de temores en condiciones de cuarentena y aislamiento social, en una muestra de adultos de la ciudad de lima, 2020. Educa UMCH, 1(15), 91100.

del Castillo, J. A. G. (2012). Concepto de percepción de riesgo y su repercusión en las adicciones. Salud y drogas, 12(2), 133-151. 
Estrella, E. C. M. (2020). Uso de personajes y metáforas en la gestión de la crisis sanitaria del COVID-19. Revisión de la comunicación de sanidad pública en España y México. Revista española de Comunicación en Salud, 319-327. https://doi.org/10.20318/recs.2020.5458

Galindo-Vázquez, O., Ramírez-Orozco, M., Costas-Muñiz, R., Mendoza-Contreras, L. A., Calderillo-Ruíz, G., \& MenesesGarcía, A. (2020). Síntomas de ansiedad, depresión y conductas de autocuidado durante la pandemia de COVID-19 en la población general. Gaceta Médica, 156, 298-305.

https://doi.org/10.24875/GMM.20000266

Gonzáles, S. V. (2019). Prácticas sexuales y percepción de riesgo frente al $\mathrm{VIH} /$ SIDA en estudiantes de la Institución Educativa Santa Rita de Cassia de San Vicente de Cañete, 2019.

González, F. A. I. (2020). Enfermedad del coronavirus 2019: la importancia del distanciamiento social. Medicina (Ribeirao Preto), 53(3), 223-233.

https://doi.org/10.11606/issn.2176-7262. v53i3p223-233

González, V., González, N., Gómez, C., Palacio, C. A., Gómez, A., \& Franco, O. H. (2020). Proyecciones de impacto de la pandemia COVID-19 en la población colombiana, según medidas de mitigación. Datos preliminares de modelos Epidemiológicos Para El Periodo Del 18 De Marzo Al 18 De Abril De 2020. Revista De Salud Pública, 22(1). https://doi.org/ 10.15446/rsap.v22n2.85789
Green, E. C., Murphy, E. M., \& Gryboski, K. (2020). The Health Belief Model. The Wiley Encyclopedia of Health Psychology, 211-214.

https://doi.org/ 10.1002/9781119057840.ch68

Diez, F. Uribe, M., Waich, A., Ospina, L., Marroquín, A., Castro, S., Patiño, J., Ariza, K. \& Gómez, C. (2020). Salud mental y resiliencia en adultos jóvenes de Sudamérica durante el aislamiento (distanciamiento social obligatorio) por la pandemia de COVID-19. Departamento de Psiquiatría y Salud Mental Departamento de Epidemiología Clínica y Bioestadística. Facultad de Medicina, Pontificia Universidad Javeriana.

https://medicina.

javeriana.edu.co/documents/3185897/0/ $\mathrm{ESM}+\mathrm{J} 0 \% \mathrm{C} 2 \% \mathrm{BFvenes+-+Prese}$ $\mathrm{nt}+$ MinSalud+versi\% C 3\%B $3 \mathrm{n}+3$. pdf/c5414e93-e078-4e0a-971f99dfd9adbdbd

Kouhpayeh, A., Jeihooni, A. K., Kashfi, S. H., \& Bahmandoost, M. (2017). Effect of an educational intervention based on the model of health beliefs in self-medication of Iranian mothers. Investigación $y$ educación en enfermería, 35(1), 59-68. https://doi.org/ 10.17533/udea.iee.v35n1a07

Lau, J. T., Griffiths, S., Choi, K. C., \& Tsui, H. Y. (2010). Avoidance behaviors and negative psychological responses in the general population in the initial stage of the H1N1 pandemic in Hong Kong. BMC Infectious Diseases, 10(1), 139.

https://doi.org/ 10.1186/1471-2334-10-139 
Medina, S., \& León, J. M. (2004). Modelos explicativos de la psicología de la salud. Psicología de la salud y calidad de vida, 61-95.

Ministerio de Educación Nacional. (2020). Lineamientos para la prestación del servicio de educación en casa y en presencialidad bajo el esquema de alternancia y la implementación de prácticas de bioseguridad en la comunidad educativa. Especial Covid-19.

https://www.mineducacion.gov.co/ 1759/w3-article-399094.html? noredirect $=1$

Muñoz, J. (2020). COVID-19: El miedo, el efecto silencioso de las epidemias. Interdisciplinary Journal of Epidemiology and Public Health, 3(1). https://doi.org/10.18041/2665-427X/ ijeph.1.6256

Ochoa-Rosales, C., González-Jaramillo, N., Vera-Calzaretta, A., \& Franco, O. H. (2020). Impacto de diferentes medidas de mitigación en el curso de la pandemia de COVID-19 en Chile: proyección preliminar para el período del 14 de abril al 14 de mayo. Revista de Salud Pública, 22(2).

https://doi.org/

10.15446/rsap.v22n2.86380

Ortiz-Hernández, L., \& Pérez-Sastré, M. A. (2020). Inequidades sociales en la progresión de la COVID-19 en población mexicana. Revista Panamericana de Salud Pública, 44.

https://doi.org/10.26633/RPSP.2020.106
Otoya, A. M., García, M. A., Jaramillo, C., \& Mahecha, Á. M. C. (2020). COVID-19: Generalidades, comportamiento epidemiológico y medidas adoptadas en medio de la pandemia en Colombia. Acta de Otorrinolaringología \& Cirugía de Cabeza y Cuello, 48(1), 93-102. https://doi.org/10.37076/acorl.v48i1.519

Pérez, K. J. B., \& Durán, M. C. B. (2020). COVID-19: Frente al desafío pedagógico de lo presencial a lo virtual. HAMUT'AY, 7(2), 9-17.

Rosabal, E. P., Sánchez, Y. M. S., Rosabal, R. P., Arias, E. L., \& Rodríguez, V. V. L. (2016). Conocimientos sobre $\mathrm{VIH} /$ sida, percepción de riesgo y comportamiento sexual en estudiantes universitarios. Multimed, 20(1).

Saura, S., Jorquera, V., Rodríguez, D., Mascort, C., Castella, I., \& García, J. (2019). Percepción del riesgo de infecciones de transmisión sexual/VIH en jóvenes desde una perspectiva de género. Atención Primaria, 51(2), 61-70. https://doi.org/

10.1016/j.aprim.2017.08.005

Valencia, C., Londoño, C., Amézquita, M., Cortés, J., Guerra, M., Hurtado, A. y Ordoñez, J. (2009). Diseño del cuestionario de creencias referidas al consumo de alcohol para jóvenes universitarios. Diversitas, 5 (2), 337-347.

https://doi.org/ 10.15332/s1794-9998.2009.0002.09 
Zimolong B, Trimpop R. (2012) Percepción del riesgo. En: OIT. Enciclopedia de Salud y Seguridad en el Trabajo., Capítulo 59.

OIT;. p. 59.25 .

https://www.insst.es/ documents/94886/162520/

Sumario+del+Volumen+

II/2e8604fd-2b20-4982-9808-

ad71b6469914 